This is an electronic reprint of the original article. This reprint may differ from the original in pagination and typographic detail.

\author{
Author(s): Geldhof, Sarina; El Youbi, Sara; Moore, lain; Pohjalainen, Ilkka; Sonnenschein, V.; \\ Terabayashi, R.; Voss, Annika
}

Title: Development of a saturated absorption spectroscopy setup at IGISOL for characterisation of Fabry-Pérot interferometers

Year: $\quad 2017$

Version:

Please cite the original version:

Geldhof, S., El Youbi, S., Moore, I., Pohjalainen, I., Sonnenschein, V., Terabayashi, R., \& Voss, A. (2017). Development of a saturated absorption spectroscopy setup at IGISOL for characterisation of Fabry-Pérot interferometers. Hyperfine Interactions, 238(1), Article 7. https://doi.org/10.1007/s10751-016-1385-3

All material supplied via JYX is protected by copyright and other intellectual property rights, and duplication or sale of all or part of any of the repository collections is not permitted, except that material may be duplicated by you for your research use or educational purposes in electronic or print form. You must obtain permission for any other use. Electronic or print copies may not be offered, whether for sale or otherwise to anyone who is not an authorised user. 


\title{
Development of a saturated absorption spectroscopy setup at IGISOL for characterisation of Fabry-Pérot interferometers
}

\author{
S. Geldhof - S. El Youbi - I. D. Moore * \\ I. Pohjalainen - V. Sonnenschein * R. Terabayashi * \\ A. Voss
}

Received: date / Accepted: date

\begin{abstract}
A saturated absorption spectroscopy setup was developed and optimised for the characterisation of a home-built and a commercial Fabry-Pérot interferometer (FPI). The free spectral range of these FPIs has been determined with reliable statistical and systematic errors. These FPIs will be used for accurate wavelength determination of broad- and narrowband pulsed Ti:Sapphire lasers used in resonance ionisation spectroscopy experiments.
\end{abstract}

Keywords Fabry-Pérot interferometer · saturation spectroscopy · rubidium

\section{Introduction}

Laser resonance ionisation offers outstanding opportunities to combine selectivity and sensitivity with an on-line ion source for means of element-selective ionisation of short-lived exotic nuclei or for the study of part-per-million pertubations in the atomic levels through measurements of hyperfine structure and isotope shifts [1]. This method of spectroscopy is commonly referred to as in-source resonance ionisation spectroscopy (RIS) and has been demonstrated both in hot cavity ion sources as well as buffer gas cells. Despite the impressive sensitivity to nuclei produced far from stability, it often remains a challenge to analyse the lower resolution in-source spectra (a reduction in spectral resolution due to environmental effects as well as the pulsed laser linewidth) and to reliably assign systematic uncertainties. In these experiments it is not possible to keep the laser frequency fixed and use a doppler tuning voltage for scanning, as in high-resolution collinear laser spectroscopy, thus the laser frequency itself must be scanned. This necessitates accurate wavelength determination. At the IGISOL facility, Jyväskylä, the wavelength of the pulsed Ti:sapphire laser radiation has been traditionally measured using a HighFinesse WS/6-UV wavemeter with an absolute accuracy of $600 \mathrm{MHz}$. Although this resolution is satisfactory for laser resonance ionisation, it was found to be unsuitable when used for spectroscopy through a measurement

S. Geldhof · S. El Youbi · I. D. Moore · I. Pohjalainen · A. Voss

Department of Physics, University of Jyväskylä, Survontie 9, PB 35 (YFL), 40014 Jyväskylä, Finland.

E-mail: sarina.m.geldhof@jyu.fi 


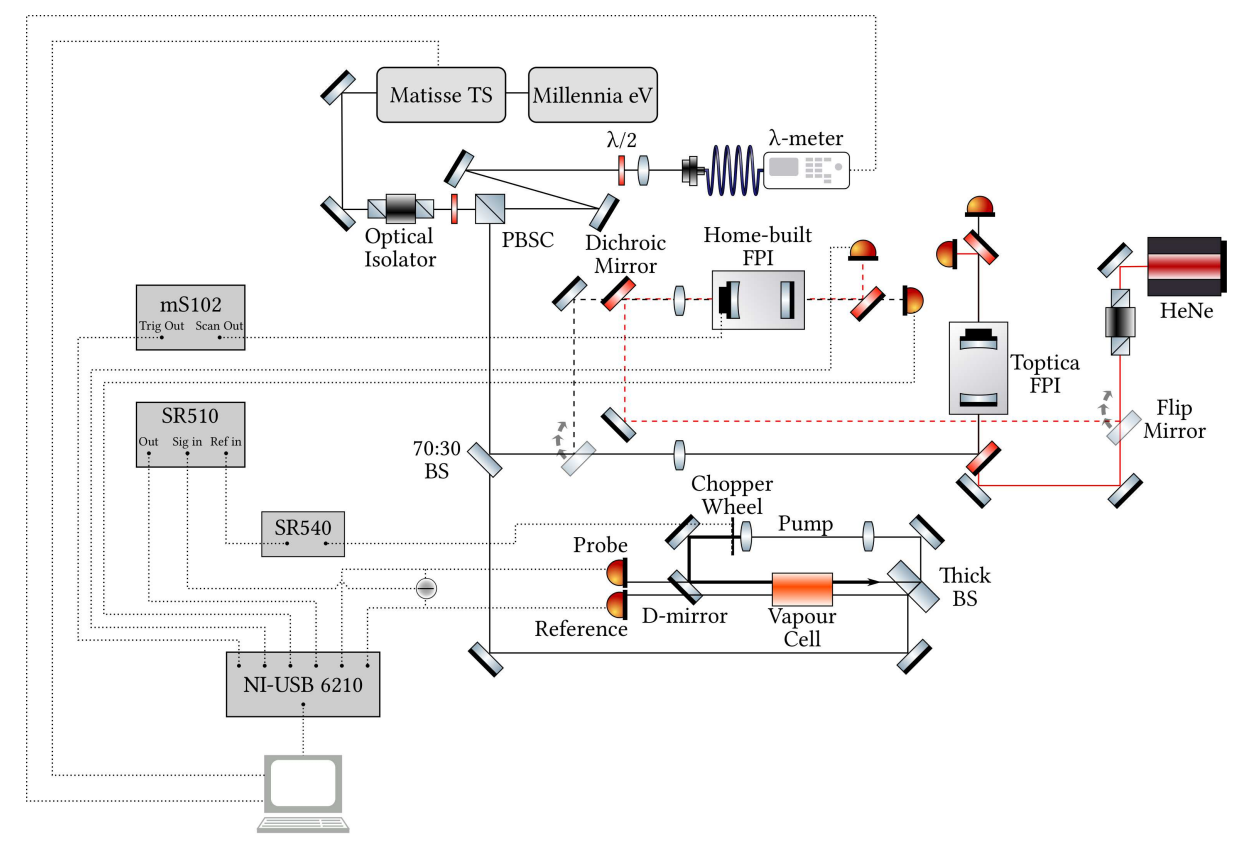

Fig. 1: Schematic of the cw laser system, the saturation absorption spectroscopy setup and two Fabry-Pérot interferometers. The ramp generator (Miniscan 102) and photodiodes can be connected to either FPI unit as required. The relevant data acquisition system is also highlighted. Colour on-line.

of the hyperfine parameters of ${ }^{63} \mathrm{Cu}$ which revealed discrepancies to existing high-resolution literature data [2].

A more appropriate method of frequency determination is with a scanning Fabry-Pérot interferometer (FPI). At IGISOL, pulsed Ti:Sapphire lasers with a broad range of bandwidths are in use. The standard resonator configuration is based on the Z-shaped standing wave design with a bandwidth of typically $5 \mathrm{GHz}$, currently in use at many RIB facilities for in-source resonance laser ionisation [3], a dual-etalon Ti:Sapphire laser for in-gas-cell spectroscopy with a bandwidth reduced to $<1 \mathrm{GHz}$ [2] and an injection-locked Ti:Sapphire laser with a narrow bandwidth of $\sim 20 \mathrm{MHz}[4,5]$, motivated for the exploitation of in-gasjet spectroscopy. These various bandwidths require FPIs with different free spectral ranges (FSRs) in order to calculate the frequency changes in a suitable mode of resolution. To measure frequency changes during scanning the interferometers must first be calibrated, in other words the FSR needs to be determined as precisely as possible. The method described in this work is via a calibration against an atomic transition exhibiting a well-known hyperfine structure. For this purpose we have developed a Doppler-free saturated absorption spectroscopy setup which uses either a Rb or Cs vapour cell, installed in a newly constructed continuous wave (cw) Ti:Sapphire laser laboratory. 


\section{Experimental setup and data acquisition}

A schematic of the saturated absorption spectroscopy setup is given in Fig. 1. The cw Ti:Sapphire laser beam (Sirah Matisse TS), pumped by a 6W Millennia eV DPSS laser, is split up in two beams by a polarising beam splitter cube (PBSC) and a half-waveplate. One of the beams is fibre coupled to the HighFinesse WS/6-UV wavemeter, the other is reflected to the experimental setup. A 70:30 beam splitter sends $70 \%$ of this beam to be used for spectroscopy with the remaining $30 \%$ delivered to the FPIs.

As can be seen in Fig. 1, in order to perform saturated absorption spectroscopy a part of the laser beam is split in two by reflecting it on the front and back surface of an uncoated thick $(12 \mathrm{~mm})$ glass beam splitter. The beam from the front reflection is termed the reference beam and the back reflection the probe beam. Both beams have a diameter of approximately $1 \mathrm{~mm}$ and pass through a vapour cell containing either natural rubidium $\left(72.168 \%{ }^{85} \mathrm{Rb}\right.$ and $27.835 \%{ }^{87} \mathrm{Rb}$ ) or caesium $\left({ }^{133} \mathrm{Cs}\right)$. Individual photodiodes (Thorlabs, FDS100) are used to record the probe and reference beam, the signals then being fed into the data acquisition. The difference between the probe and reference signals is generated electronically and used as an input for the lock-in amplifier (Stanford Research Systems, SR510). The majority of the laser light, however, is transmitted by the thick beam splitter and becomes the pump beam, after expansion to a larger diameter of about $3 \mathrm{~mm}$ using a telescope. Before being overlapped in a counter-propagating direction with the probe beam by reflection from a D-shaped mirror, the pump beam is modulated using a chopper wheel. This wheel can be operated at different frequencies set by the chopper controller (Stanford Research Systems, SR540) and is needed in conjunction with the lock-in amplifier.

In addition to the saturated absorption spectroscopy setup, $30 \%$ of the Ti:Sapphire beam is sent to one of two scanning FPIs together with a frequency-stabilised HeNe laser (Melles Griot, 25-STP-912-230), stable to within a MHz per hour and $10 \mathrm{MHz}$ per month according to specifications [6]. One FPI is home-built and uses a semi-hemispherical geometry, with a planar and a concave mirror $(7.5 \mathrm{~cm}$ radius of curvature) having a broadband coating $(97 \%$ reflectivity for 630-1050 nm) [7]. Due to the non-confocal nature of the FPI the laser beams must be mode-matched to the cavity in order to suppress higher order Hermite-Gaussian modes. The incoming laser beams may also have different waist sizes which will affect the mode-matching, and thus both beams pass through an aperture of approximately $1 \mathrm{~mm}$ in diameter before being focused into the FPI. Currently the finesse is limited to a value of $\sim 75$, however values of close to 1500 were reached when using a pair of high reflecting mirrors of $\mathrm{R}=99.8 \%$, at the expense of greatly reduced transmission. The second FPI is a confocal commercial Toptica FPI-100-0750-y with an FSR of 1 GHz and a Finesse of 200-500 with the mirror set coated for 615-885 nm [8]. For both FPIs, scanning of one mirror by a piezo is provided by a ramp generator (Toptica, Miniscan 102). The Ti:Sapphire and HeNe beams are combined and split up again after the FPI with dichroic mirrors (Thorlabs, M254C45) and are detected on photodetectors with variable amplification (Thorlabs, PDA36A-EC). Fast switching between the FPIs is provided by flip mirrors.

The data acquisition hardware module from National Instruments (NI USB-6210) has 16 analogue voltage input channels with a sampling rate of $250 \mathrm{kS} / \mathrm{s}$ shared between all channels in use [9]. In this work, six signals are connected: the photodiode signals from both probe and reference beam, the FPI photodiode signals of the HeNe and the Ti:Sapphire, the lockin amplifier output and the trigger signal of the ramp generator. Acquisition and on-line visualisation is controlled in LabVIEW ${ }^{\mathrm{TM}}$. The DAQ sampling rate puts a maximum limit on the piezo scan speed of the high finesse FPI cavities, as proper peak detection is needed. 

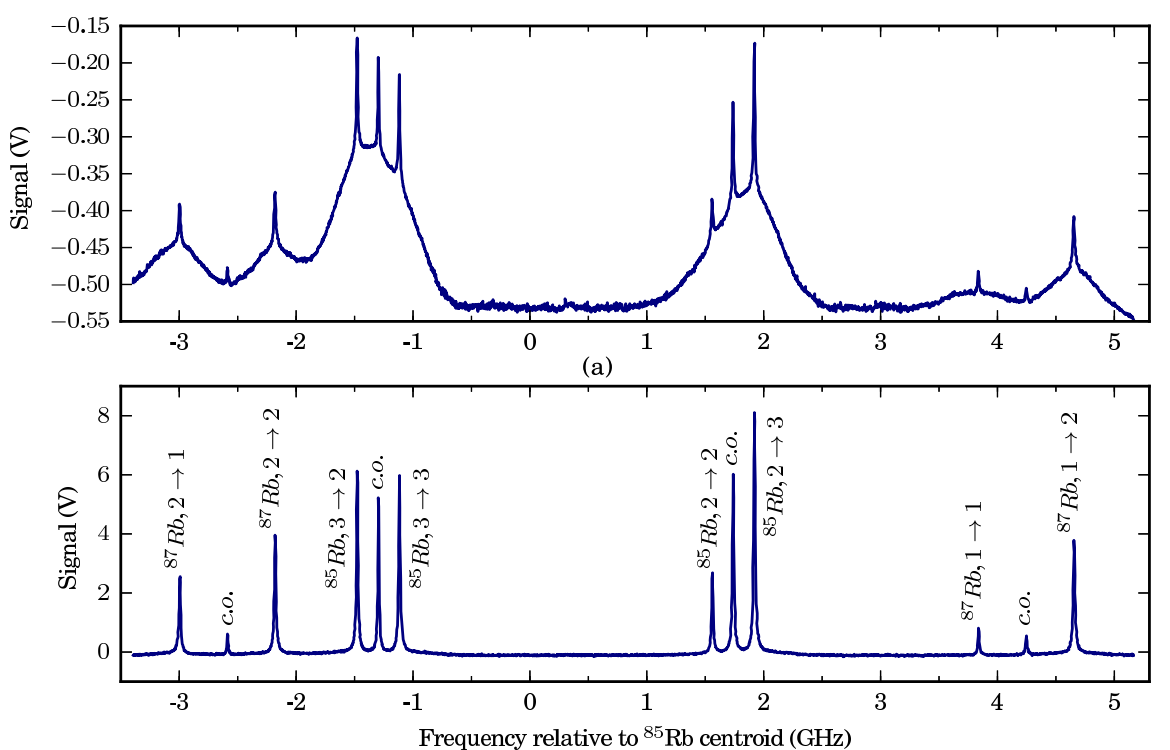

(b)

Fig. 2: (a) The direct difference between probe and reference on the Rb D1 transition. (b) The spectrum after the lock-in amplifier. The residual Doppler-broadened background is removed, the noise is reduced and the resonances are amplified. Labels indicate the transitions, with c.o. used for the cross-over resonances.

Since the raw data of all signals would quickly reach an inconvenient file size, real-time analysis is used for data reduction. A time average over one FPI scan is taken for the probe and reference signals. A linear fit of the data over this period of one FPI scan is performed to calculate the error using the fit residuals. Only the average of each signal and the standard deviation of the fit residuals are saved. By utilising a peak detection routine in LabVIEW ${ }^{\mathrm{TM}}$, only the location and amplitude of the transmission fringes are recorded. The acquisition and analysis loops are separated in order to operate simultaneously with optimal performance. The peak detection is performed using the default peak detect function in LabVIEW ${ }^{\mathrm{TM}}$, Peak detector VI [10]. It is based on an algorithm that fits a quadratic polynomial to consecutive groups of data points specified by an adjustable interval.

\section{Optimisation of saturated absorption setup}

According to saturated absorption theory [11], it is possible to obtain a Doppler-free spectrum with a flat background and a spectral linewidth only limited by the natural linewidth of the atomic transition by subtracting the reference signal from the probe signal, as all fluctuations should be identical for the two beams. In the normal experimental setup without a lock-in amplifier a background noise signal was always seen. This was due to imbalances in the photodiodes and their amplifiers, and absorption differences in the probe and reference beam. The reflections of the thick beam splitter are not equal in power, so slight steering and power changes while scanning the Ti:sapphire laser frequency affect both photodiode 

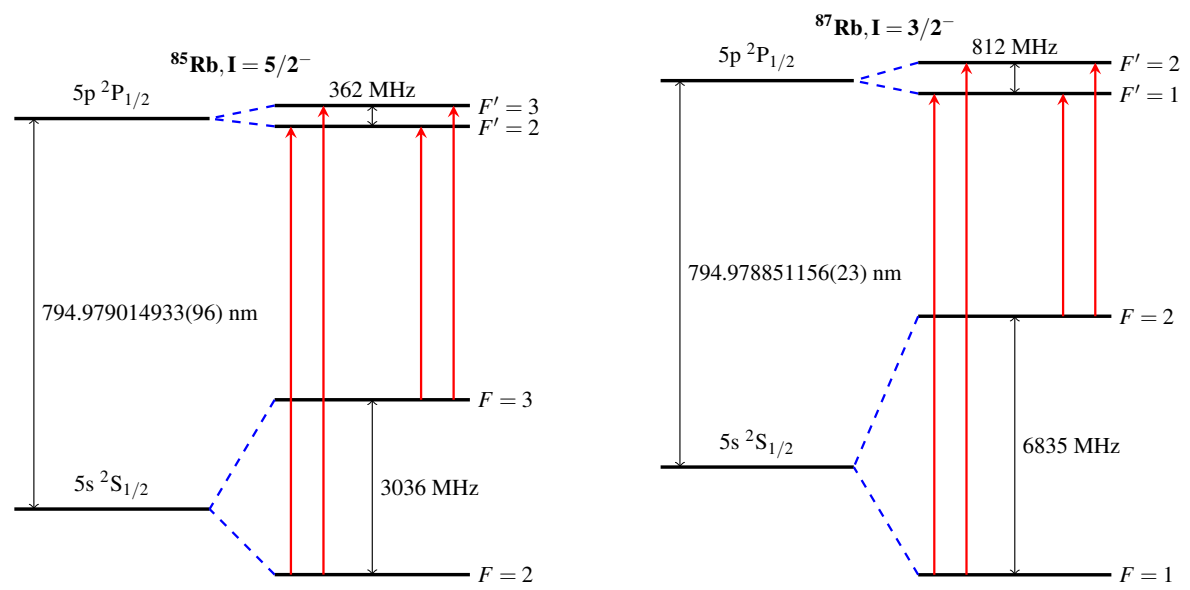

Fig. 3: D1 transition in rubidium with wavelength in vacuum and hyperfine splittings in MHz. Allowed transitions are indicated by arrows. The HFS splittings were calculated using the standard HFS equation [12] using HFS parameters from [13,14].

signals differently. This can give rise to a non-constant background and residual Doppler broadening in the difference signal. An example can be seen in Fig. 2a for a spectrum on the Rb D1 transition, the details of which are presented in Fig. 3. So-called "cross-over" (c.o.) resonances are inherent to the saturated absorption spectroscopy technique when multiple hyperfine transitions are located within one Doppler broadened feature. They occur when pump and probe beam are resonant with different hyperfine transitions of the same multiplet. Fig. 2 shows four c.o. resonances in addition to the six hyperfine resonances.

To solve the problem of the residual background contributions, a lock-in amplifier was installed as shown in Fig. 1 using the direct difference between probe and reference beam as input. The waveform from the chopper controller, a square wave, is used as the reference signal for the lock-in amplifier. This eliminates the background as only signals correlated with the pump beam are recorded, and amplifies the resonances. An example of the spectral enhancement can be seen in Fig. 2b. This experimental setup was tested to obtain the optimal conditions. As the input and reference signal are mixed in the lock-in amplifier, it can be shown with simple mathematics that the output is of the form $V_{\text {out }}=\frac{1}{2} V_{\text {signal }} V_{\text {ref }} \cos (\Delta \phi)$ where $V_{i}$ are the amplitudes and $\Delta \phi$ the phase difference between the signal and reference. Therefore changing the phase angle between the input and reference signal on the lockin amplifier should yield a cosine dependence on the peak intensities as is experimentally observed in Fig. 4. Notice that the results shown in Fig. 4 are for spectroscopy on Cs.

As considerable condensation was present at room temperature in the Rb vapour cell, a heating controller (Thorlabs TC200) was installed around the cell. The effect of heating was investigated by comparing the depth of the Doppler-broadened absorption (using the single reference beam) and the saturated absorption peak intensity for different temperatures, as shown in Fig. 5. Cs on the other hand showed a very strong absorption, even at room temperatures, therefore the heater is not required in this case. 


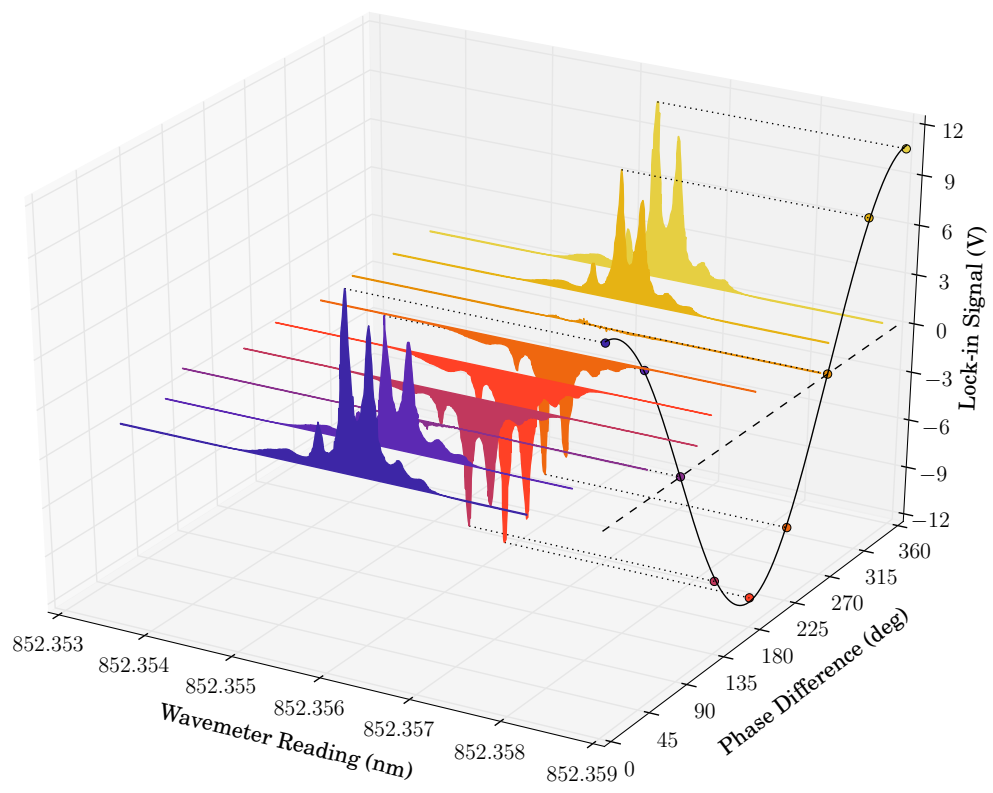

Fig. 4: Partial hyperfine spectra taken on the Cs D2 transition by changing the phase angle between input and reference signal in the lock-in amplifier. The peak intensities show a cosine dependence as expected from theory. Colour on-line.

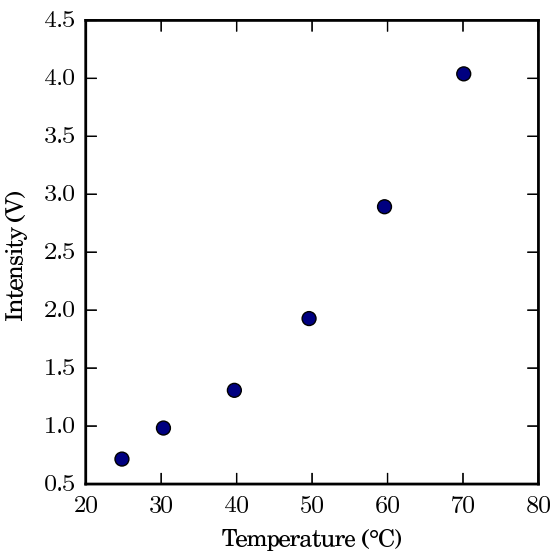

(a) Doppler depth

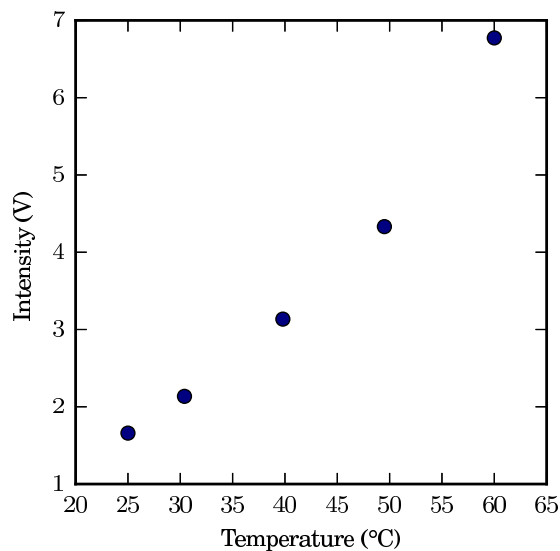

(b) Peak intensity of $\mathrm{F}=3$ to $\mathrm{F}^{\prime}=2$ transition

Fig. 5: Effect of heating the Rb vapour cell on the (a) Doppler-broadened absorption depth and (b) saturated absorption peak intensity of the $F=3$ to $F^{\prime}=2$ transition in the $D 1$ transition of ${ }^{85} \mathrm{Rb}$. Error bars are within data points. 


\section{Fabry-Pérot interferometer characterisation}

\subsection{Procedure}

In order to characterise the FSR of the FPIs, the saturated absorption spectrum of the D1 transition in $\mathrm{Rb}$ was recorded, simultaneously with the transmission fringes of both the stabilised HeNe laser and the scanning cw Ti:sapphire laser.

The initial step in the characterisation procedure is to convert the recorded time scale to a scale in number of FSR. The data is first split up into separate FPI scans using the ramp trigger signal as an indicator of the start and stop of each scan. Both the HeNe and Ti:Sapphire transmission fringe positions in a scan are then identified. Ideally, the distance between consecutive fringes should be constant, however this is not the case due to the non-linearity of the piezo scanning of the FPI mirror. Therefore, the fringe positions are linearised. The positions of the HeNe fringes, with the first fringe set to zero for reference, are plotted versus the number of fringes counted, which is equal to the number of scanned FSRs. The data points are then fitted with polynomials of different orders. An example of one such fit can be seen in Fig. 6. While the deviation between a linear fit and the data points is of the order of $5 \%$ of one FSR, the third degree polynomial reproduces the data accurate to $0.4 \%$. Higher order polynomials did not significantly improve the fit. The positions of the Ti:Sapphire fringes are then converted to the number of FSRs scanned, using the fit parameters obtained from the HeNe transmission fringes and scaling by the ratio of the wavelengths, $\lambda_{\mathrm{HeNe}} / \lambda_{\mathrm{Ti}: \mathrm{Sa}}=632.099 \mathrm{~nm} / 794.979 \mathrm{~nm}$.

Secondly, one Ti:Sapphire fringe and the corresponding HeNe fringe are chosen, to track and use for the determination of the number of FSR scale. While temperature changes and vibrations will cause drifting of the transmission fringes, both the HeNe and Ti:Sapphire are affected in the same manner. The position difference between the Ti:Sapphire and HeNe fringes is thus unaffected. As the tuning range of the Ti:Sapphire laser often spans multiple FSRs to cover the whole hyperfine structure, the tracked Ti:Sapphire fringe would at some point leave the available scanning range provided by the piezo. To overcome this problem, the transmission order of the tracked Ti:Sapphire fringe is changed by one in the analysis procedure each time the currently tracked fringe moves too far from the HeNe fringe, i.e. by more than half an FSR (Fig. 7). The scanned number of FSRs is then the value obtained from the linearisation fit procedure for the tracked Ti:Sapphire fringe plus the number of jumps, $N_{j}$, up to that point in the Ti:Sapphire scan,

$$
F S R=F S R_{f i t}+N_{j}
$$

To improve the statistical uncertainty, multiple Ti:Sapphire fringes may be tracked in the same way to calculate an average for $F S R_{f i t}$. The number of Ti:Sapphire fringes one can choose to track depends on the number of recorded Ti:Sapphire fringes per scan, the nonlinearity and the temperature stability. Usually the piezo movement is highly non-linear at the start and end of a scan, so it is best not to use the Ti:Sapphire fringes close to a change in scan direction. Temperature fluctuations will move the HeNe fringes, so some transmission orders might (dis)appear from the FPI scanning range. As the same $\mathrm{HeNe}$ transmission orders are tracked over the whole spectrum, only orders that are present throughout the entire hyperfine structure scan can be used. Based on these considerations, an optimal number of Ti:Sapphire fringes can be chosen for each spectrum.

The difference between the number of FSRs obtained for each Ti:Sapphire fringe from the fit, after rescaling by the wavelength ratio, should be equal to one, as one FSR is de- 

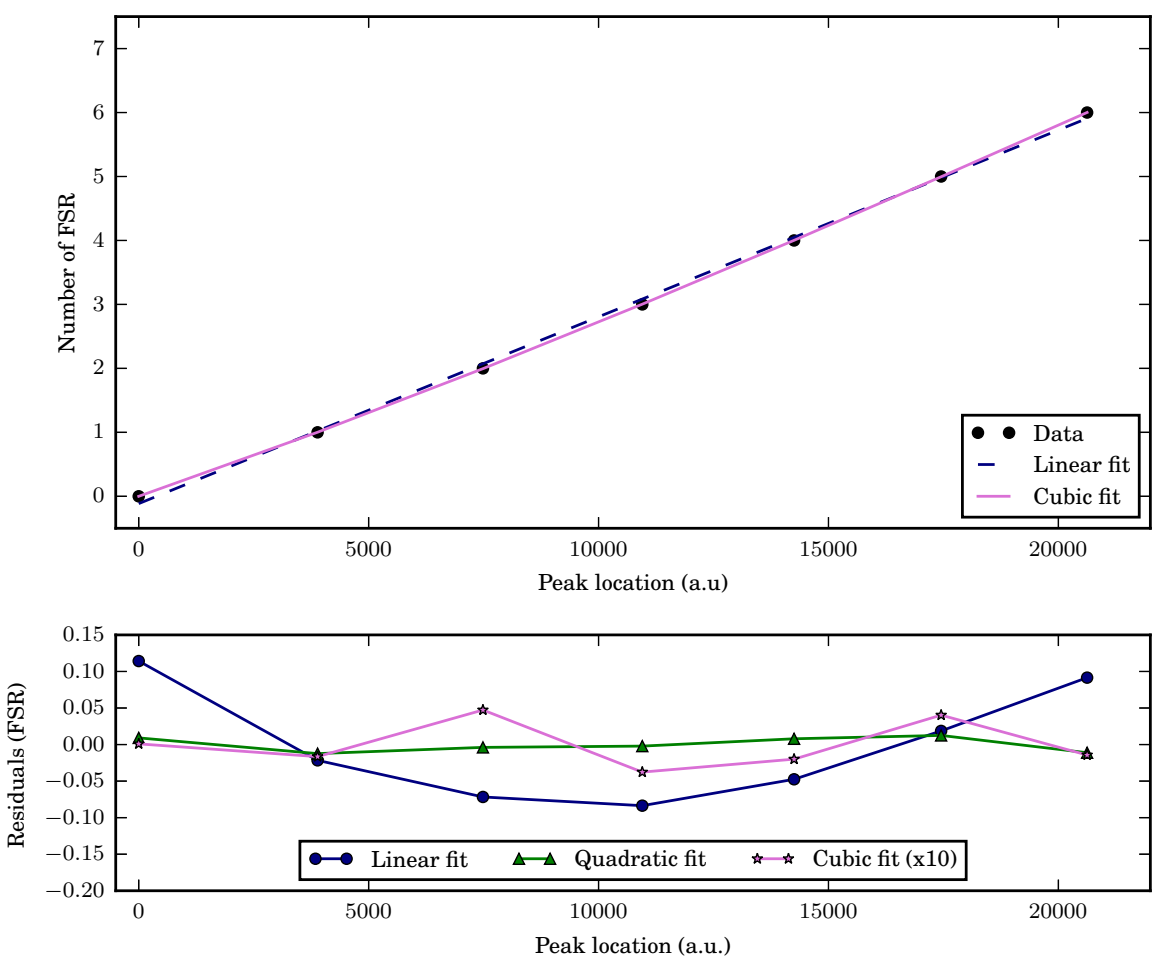

Fig. 6: An example of the linearisation of the relative HeNe fringe positions against FSR number. In the upper plot the quadratic fit is not shown for clarity. The lower plot shows the residuals for the different polynomials with the residuals of the cubic fit multiplied by ten. Colour on-line.

fined as the spacing between two fringes. The deviation from one gives the remaining nonlinearity that is not compensated. For each FPI scan the average of the differences from one is calculated, and then finally, the average of all values for one spectrum is determined. This value is added as a systematic error to the measured value of the FSR.

Combining the number of FSRs scanned with the recorded photodiode signals, a spectrum can be plotted with one data point for each FPI scan. The value of the FSR, in frequency units, is then obtained from a hyperfine structure fit with the all hyperfine parameters fixed to literature values but multiplied by a free-running common scaling factor. The inverse of this scaling factor is an accurate measure of the FSR.

\subsection{Results}

In total 14 spectra were recorded on the D1 transition in Rb, two for the home-built FPI and eight for the Toptica FPI without lock-in amplifier, and two scans for each FPI with the lock-in amplifier. Each spectrum was converted to an x-axis in number of FSR using the procedure outlined in section 4.1, each time using the optimal number of Ti:Sapphire fringes. 
Fig. 7: Illustration of the fringe tracking procedure. Dotted lines are HeNe fringes, solid lines are Ti:Sapphire fringes. The darker HeNe fringe is the reference (labeled below). The left figure represents the first FPI scan. In the figure to the right, recorded some time later, the previously tracked fringe has moved beyond the limit of half an FSR from the reference HeNe fringe, indicated by the dashed red line, and thus a jump back has occurred. Colour on-line.

Table 1: FSR values for the home-built and commercial Toptica FPI. The values per technique are weighted averages, and the final values are the weighted averages running over all individual values. Only statistical uncertainties are quoted.

\begin{tabular}{lllll}
\hline & $\begin{array}{l}\text { Without lock-in } \\
(\mathrm{GHz})\end{array}$ & $\begin{array}{l}\text { With lock-in } \\
(\mathrm{GHz})\end{array}$ & $\begin{array}{l}\text { Previous measurement } \\
(\mathrm{GHz})[5]\end{array}$ & $\begin{array}{l}\text { Final value } \\
(\mathrm{GHz})\end{array}$ \\
\hline $\begin{array}{l}\text { Toptica FPI } \\
\text { Home-built FPI }\end{array}$ & $\begin{array}{l}0.99887(4) \\
3.4765(2)\end{array}$ & $\begin{array}{l}0.998569(15) \\
3.46525(5)\end{array}$ & $0.99850(15)$ & $0.998604(14)$ \\
\hline
\end{tabular}

Hyperfine structure fits were performed to the $\mathrm{Rb}$ spectra with Lorentzian lineshapes, a common full width at half maximum (FWHM) and the common scaling factor for the hyperfine parameters and isotope shift fixed to the literature values $[13,14]$. All peak intensities were left free, as constraining them by the Racah coefficients had a negative effect due to the peak positions not being in the exact location given by the hyperfine parameters. The positions of the crossover peaks were determined using the hyperfine parameters, and thus are also scaled by the common factor.

The weighted averaged FSR values for each FPI, with and without the use of the lock-in amplifier, can be found in Table 1, including a previous value determined for the commercial Toptica FPI [5]. The values per technique are weighted averages, however the final weighted average per FPI runs over all individual values.The final adopted values for the FSRs of both FPIs are the weighted averages of the individual values. The systematic uncertainties, calculated from the remaining non-linearity as explained in section 4.1, are $4 \mathrm{MHz}$ for the Toptica FPI and $5 \mathrm{MHz}$ for the home-built FPI, respectively. Temperature drifts are the main cause of the systematic error, as the linearisation becomes less reliable when the $\mathrm{HeNe}$ fringes move considerably. The continuous wave laboratory was recently upgraded, with 


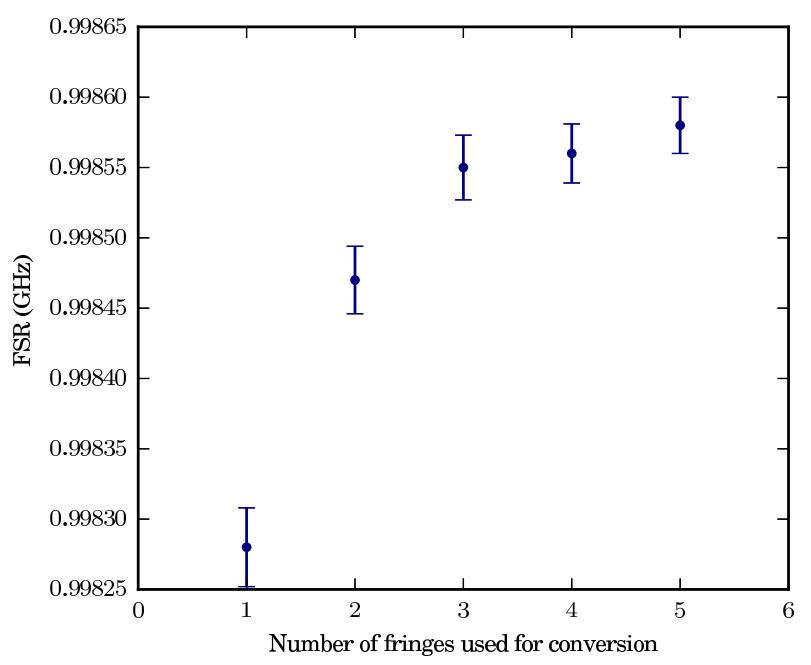

Fig. 8: Example of improvement of statistical errors (a small reduction in the size of the error bars) and convergence of the FSR value with increasing number of Ti:sapphire transmission fringes used for conversion for the Toptica FPI. Systematic errors are not included.

the optical table better shielded from the air flow from the air-conditioning unit. Very recent tests show that the systematic error for the Toptica FPI has been reduced to $3 \mathrm{MHz}$. This directly correlates to less drifting of the HeNe fringes in the new environment, as was seen when tracking the fringes over time.

To prove that using multiple Ti:Sapphire fringes for the conversion to scanned number of FSR improves the statistical uncertainty, the conversion for four spectra was done using one to five fringes out of a total of about six or seven fringes. Five fringes was the maximum possible due to temperature instabilities. For each number of used fringes the fit to determine the FSR was performed. In all spectra the reduced chi-squared improved with the number of fringes used. The statistical uncertainty on the FSR value also decreased and the value itself appears to saturate with increasing number of fringes, shown for one spectrum in Fig. 8.

As a consistency check, the four spectra taken with the lock-in amplifier were converted to an actual frequency scale. This is done by multiplying the scanned number of FSR from Eq. 1 by the obtained FSR in frequency units from Table 1. The fit to the hyperfine structure was then redone, however now using free-running hyperfine parameters and isotope shift, again with Lorentzian lineshapes, a common FWHM and free intensities. Typical differences of the hyperfine parameters with respect to literature values are of the order of 0.5 MHz for the Toptica and $0.8 \mathrm{MHz}$ for the home-built FPI, as can be seen in Table 2, which is considerably smaller than the systematic errors of either 4 or $5 \mathrm{MHz}$. This gives confidence in the assignment of the systematic errors.

\section{Conclusion}

A saturated absorption spectroscopy setup for $\mathrm{Rb}$ and $\mathrm{Cs}$ has been implemented at the IGISOL facility and optimised using phase-sensitive detection via a lock-in amplifier. Us- 
Table 2: Results of the fit with free-running hyperfine parameters and isotope shift as a consistency check. Only statistical errors are shown. None of the values deviates more than the systematic error which is dominated by temperature instabilities.

\begin{tabular}{lcrr}
\hline & $\begin{array}{l}\text { Literature } \\
(\mathrm{MHz})[13,14]\end{array}$ & \multicolumn{1}{c}{$\begin{array}{c}\text { Toptica FPI } \\
\text { (MHz) }\end{array}$} & \multicolumn{1}{l}{$\begin{array}{l}\text { Home-built FPI } \\
\text { (MHz) }\end{array}$} \\
\hline$A_{85,{ }^{2} P_{1 / 2}}$ & $120.527(56)$ & $121.34(3)$ & $120.71(3)$ \\
$A_{85,}{ }^{2} S_{1 / 2}$ & $1011.9108130(20)$ & $1011.72(3)$ & $1011.98(3)$ \\
$A_{87,}{ }^{2} P_{1 / 2}$ & $407.25(63)$ & $407.90(7)$ & $408.54(6)$ \\
$A_{87,2}{ }^{2} S_{1 / 2}$ & $3417.341305452145(45)$ & $3417.19(6)$ & $3416.76(6)$ \\
$v_{87-85}$ & $78.095(12)$ & $78.29(7)$ & $78.40(7)$ \\
\hline
\end{tabular}

ing the saturated absorption spectrum of the $\mathrm{D} 1$ transition in $\mathrm{Rb}$ as a calibration, a procedure for frequency conversion using FPIs has been developed and described in detail. The free spectral range of a commercial Toptica and a home-built FPI were determined to be $0.998836(13)[4000] \mathrm{GHz}$ and 3.46571(5)[500] GHz, respectively. The technique has resulted in a good understanding of statistical and systematic errors.

The rather large size of the systematic uncertainties (of the order of a few $\mathrm{MHz}$ ) is most likely due to temperature instabilities within the laser laboratory. Work on the general lab environment has been undertaken to reduce the uncertainties further and investigations to quantify the instabilities are currently of high priority. In order to mitigate any temperature drifts in the future, one route to be pursued will be to temperature stabilise the FPIs well above room temperature in order to be independent from the general laboratory environment.

Improvements are also underway for the data acquisition. It has been observed that small fluctuations in the FPI photodiode signals may cause errors such as duplicate detection in the peak detector routine. As the transmisson fringes can be expressed as series of Lorentzian functions [15], a new method based on Lorentzian fitting is expected to yield a more reliable and accurate result. It is further anticipated to interface the DAQ system with the scanning of the laser through the Matisse Commander such that individual frequency steps can be taken.

In the future, the cw Ti:sapphire laser will be used for high-resolution collinear laser spectroscopy experiments and thus will require long-term frequency stabilisation. Typical stabilisation methods include the use of a suitable commercial wavelength meter [16] or by transferring the stability of a master laser via an FPI of low FSR [17]. Often, a frequency stabilised HeNe laser acts as the master laser achieving a long-term frequency stability of $\sim 400 \mathrm{kHz}$ relative to the $\mathrm{HeNe}$ [18]. It is foreseen to investigate the frequency stability of the HeNe laser used in the experiments presented here by stabilising the Matisse TS against a saturated absorption peak and tracking the transmission fringes of both lasers via a $150 \mathrm{MHz}$ FPI. In parallel, tendering for a new commerical wavemeter has begun. The best method of frequency stabilising the cw Ti:Sapphire laser will then be evaluated.

Acknowledgements This work has been supported by the Academy of Finland programme under the Finnish Centre of Excellence Programme 2012-2017 (Project No. 251353, Nuclear and Accelerator-Based Physics Research at JYFL). 


\section{References}

1. Fedosseev, V.N., Kudryavtsev, Y., Mishin, V.I.: Resonance laser ionization of atoms for nuclear physics. Physica Scripta 85(5), 058,104 (2012)

2. Sonnenschein, V., Moore, I., Khan, H., Pohjalainen, I., Reponen, M.: Characterization of a dual-etalon Ti:Sapphire laser via resonance ionization spectroscopy of stable copper isotopes. Hyp. Int. 227(1-3), 113-123 (2014)

3. Geppert, C.: Laser systems for on-line laser ion sources. Nucl. Instrum. Meth. B 266(19-20), $4354-$ 4361 (2008). Proceedings of the XVth International Conference on Electromagnetic Isotope Separators and Techniques Related to their Applications

4. Kessler, T., Tomita, H., Mattolat, C., Raeder, S., Wendt, K.: An injection-seeded high-repetition rate Ti:Sapphire laser for high-resolution spectroscopy and trace analysis of rare isotopes. Laser Phys. 18(7), 1-8 (2008)

5. Sonnenschein, V.: Laser developments and high resolution resonance ionization spectroscopy of actinide elements. Ph.D. thesis, University of Jyväskylä (2014)

6. Melles Griot: Specifications stabilized helium neon laser system 05-STP-912

7. Khan, H.: Fabry-Perot interferometer; construction, calibration and development. Master's thesis, University of Jyväskylä (2015)

8. TOPTICA Photonics AG: FPI 100 - Fabry-Perot Interferometer. http://www.toptica.com/products/ photonicals/scanning_fabry_perot_interferometer_and_detector_unit

9. National Instruments: NI USB-621x User Manual (2009)

10. National Instruments: LabVIEW 2012 Help - Peak Detector VI (2012). https://zone.ni.com/reference/en-

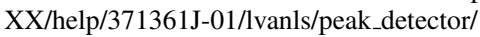

11. Demtröder, W.: Laser Spectroscopy 2: Experimental techniques. Springer (2008)

12. Campbell, P., Moore, I., Pearson, M.: Laser spectroscopy for nuclear structure physics. Prog. Part. Nucl. Phys. 86, 127-180 (2016)

13. Steck, D.A.: Rubidium 85 D line data. http://steck.us/alkalidata. (revision 2.1.6, 20 September 2013)

14. Steck, D.A.: Rubidium 87 D line data. http://steck.us/alkalidata. (revision 2.1.5, 13 January 2015)

15. Henderson, A.R.: Fabry-Perot interferometers. G. Hernandez Cambridge University Press. Opt. Laser Technol. 18, 274 (1986)

16. Minamisono, K., Mantica, P., Klose, A., Vinnikova, S., Schneider, A., Johnson, B., Barquest, B.: Commissioning of the collinear laser spectroscopy system in the BECOLA facility at NSCL. Nucl. Instrum. Meth. A 709, 85-94 (2013)

17. Zhao, W., Simsarian, J., Orozco, L., Sprouse, G.: A computer-based digital feedback control of frequency drift of multiple lasers. Rev. Sci. Instrum. 69(11), 3737-3740 (1998)

18. Voss, A., Procter, T., Shelbaya, O., Amaudruz, P., Buchinger, F., Crawford, J., Daviel, S., Mané, E., Pearson, M., Al Tamimi, W.: The Collinear Fast Beam laser Spectroscopy (Cfbs) experiment at Triumf. Nucl. Instrum. Meth. A 811, 57-69 (2016) 